\title{
Classificação automática de glóbulos brancos usando descritores de forma e textura e eXtreme Gradient Boosting
}

\author{
Domingos A. Dias Júnior ${ }^{1}$, Luana B. da Cruz ${ }^{1}$ João O. B. Diniz ${ }^{1,2}$, \\ Geraldo Braz Júnior ${ }^{1}$ Aristófanes C. Silva ${ }^{1}$ \\ ${ }^{1}$ Núcleo de Computação Aplicada - Universidade Federal do Maranhão (UFMA) \\ Caixa Postal 65.085-580 — São Luís - MA — Brasil \\ ${ }^{2}$ Instituto Federal de Educação, Ciência e Tecnologia do Maranhão (IFMA) \\ Caixa Postal 65.940-000 — Grajaú - MA — Brasil \\ \{domingos.adj, luana.b.cruz, geraldo\}@nca.ufma.br,
joao.bandeira@ifma.edu.br
}

\begin{abstract}
Blood diseases diagnosis involves the identification and characterization of blood samples from patients by counting and classifying white blood cells. Automated methods have important applications for assisting doctors. The goal of this work is to develop a method for automatic classification of white blood cells using enhancement techniques, Threshold Adjacency Statistics (TAS) for the feature extraction and eXtreme Gradient Boosting (XGBoost) for classification. The results are promising compared to other techniques and works in the literature, reaching $93.27 \%$ accuracy and $90 \%$ F-Measure. With this, it is believed that the method can assist specialists in this important task.
\end{abstract}

Resumo. $O$ diagnóstico de doenças sanguíneas envolve a identificação $e$ caracterização de amostras de sangue de pacientes pela contagem $e$ classificação de glóbulos brancos. Métodos automatizados têm importantes aplicações para auxiliar médicos. O objetivo deste trabalho é desenvolver um método para classificação automática de glóbulos brancos utilizando técnicas de realce, Threshold Adjacency Statistics (TAS) para extração de características e eXtreme Gradient Boosting (XGBoost) para classificação. Os resultados são promissores comparados a outras técnicas e trabalhos da literatura, alcançado 93,27\% de acurácia e 90\% de F-Measure. Com isto, acredita-se que o método possa auxiliar especialista nesta tarefa importante.

\section{Introdução}

Os glóbulos brancos são um tipo de célula sanguínea que é produzido na medula óssea e é encontrado no sangue e no tecido linfático. Estes fazem parte do sistema imunológico do corpo e ajudam o corpo a combater infecções e outras doenças. Os tipos de glóbulos brancos são neutrófilos, eosinófilos, basófilos, monócitos e linfócitos [NCI 2020, ACS 2018].

O diagnóstico de doenças sanguíneas geralmente envolve a identificação e caracterização de amostras de sangue de pacientes. Entre eles, a contagem e classificação de glóbulos brancos, pois auxiliam os patologistas a diagnosticar doenças como leucemia e outras [NCI 2020]. Métodos manuais ou automáticos são frequentemente usados para 
classificar os glóbulos brancos. Embora o método manual possa alcançar uma alta taxa de reconhecimento de células sanguíneas, existem desvantagens como análise lenta, precisão não padronizada e alta dependência das habilidades dos especialistas [Zhao et al. 2017].

Atualmente, métodos automatizados de detecção e classificação de tipos de células sanguíneas têm importantes aplicações médicas, como os trabalhos recentes de [Vogado et al. 2019, Mohamed et al. 2020, Vatathanavaro et al. 2018]. Esses sistemas aumentam a eficiência da análise e reduzem a carga de trabalho dos médicos especialistas. Portanto, métodos automáticos para a classificação de glóbulos brancos são cada vez mais úteis no processo de detecção e diagnóstico de doenças no sangue.

Assim, o objetivo deste trabalho é apresentar um método automático para classificação de glóbulos brancos em imagens microscópicas, utilizando técnicas de melhoramento de imagem, extração de características baseadas em Threshold Adjacency Statistics (TAS) e o classificador eXtreme Gradient Boosting (XGBoost). O método proposto poderá ser incorporado a um sistema do tipo CADx (Computer Aided Diagnosis) e, portanto, contribuir para o aumento da produtividade e melhoria nas taxas de diagnóstico.

Por propor um método completamente automático e inovador, o mesmo alcança uma série de contribuições, das quais pode-se destacar: (1) um método totalmente automatizado para classificar imagens microscópicas em quatro classes (eosinófilos, linfócitos, monócitos e neutrófilos); (2) proposição de técnicas de melhoramento de imagem para destacar as características das imagens de glóbulos brancos; (3) desenvolvimento um método capaz de extrair características de imagens microscópicas; e (4) método que usa o XGBoost como um classificador para características profundas.

Além desta seção, este artigo ainda apresentará mais quatro seções. A Seção 2 descreve os trabalhos mais recentes relacionados à classificação de glóbulos brancos. A Seção 3 apresenta e detalha o método proposto. Os resultados e discussões serão apresentados na Seção 4. Por fim, na Seção 5 são destacadas as conclusões e trabalhos futuros.

\section{Trabalhos Relacionados}

A classificação de glóbulos brancos em imagens microscópicas é um campo de pesquisa amplamente estudado. Nesta seção, serão apresentados alguns destes trabalhos.

No trabalho de [Rezatofighi and Soltanian-Zadeh 2011] é apresentado um método para classificação de glóbulos brancos usando extração de características baseada em morfologia e textura em conjunto com redes neurais artificiais e Máquina de Vetores de Suporte. O método é testado em 5 classes de glóbulos brancos divididos em 400 imagens, e obteve resultados de 93,09\% de acurácia média. Em [Ko et al. 2011] faz a extração de características de textura, cor e forma resultando em 71 características para a classificação de 200 imagens de glóbulos brancos divididos em 5 classes. Na etapa de classificação, os autores utilizam o algoritmo Random Forest apresentando uma precisão de 72,5\% e sensibilidade de $67,34 \%$.

Por sua vez, [Tai et al. 2011] propõe um método baseado em Máquina de Vetores de Suporte para a classificação de glóbulos brancos. Os autores utilizam características geométricas em conjunto com o classificador em 210 imagens de 7 
classes, obtendo resultado médio de sensibilidade e precisão de 95,3\% e 95,27\%, respectivamente. Em [Macawile et al. 2018] é apresentado um método baseado em redes neurais convolucionais (Convolutional Neural Network - CNN) para classificar 5 classes de glóbulos brancos divididos em 178 imagens. O método treina 3 diferentes CNNs (AlexNet, GoogLeNet, ResNet) e classifica as imagens de teste. O trabalho apresenta mais de $95 \%$ de acurácia média para as classes em todas as CNNs. No trabalho de [Vatathanavaro et al. 2018] apresenta a comparação entre duas técnicas de aprendizado profundo, a VGG e a ResNet para classificar 5 classes de glóbulos brancos em 810 imagens. Os experimentos mostram que a ResNet apresenta os melhores resultados, atingindo uma acurácia média de 88,29\%, enquanto a VGG apenas 72,07\%.

Por fim, [H Mohamed et al. 2020] propuseram um método de aprendizado profundo para classificação de glóbulos brancos. Uma abordagem excluindo a última camada da CNN e usando a saída da penúltima como características foi sugerida, usando 7 classificadores com características extraídas de CNNs. O método foi aplicado em uma base de dados de 9760 imagens, e os melhores resultados foram obtidos com a arquitetura CNN DenseNet com regressão logística, apresentando uma acurácia média de 92\%, Fscore de $86 \%$ e precisão de $86 \%$.

Assim, observa-se que trabalhos que buscam a classificação de glóbulos brancos ainda são muito estudados. A fase de extração de características de imagem é uma tarefa árdua que requer um alto nível de especialização e, portanto, considerável tempo e esforço. Métodos de aprendizado profundo vêm ganhando bastante espaço nos últimos anos. Todavia, estes métodos são custosos e a criação de um modelo ideal e genérico nem sempre é uma tarefa trivial.

Outro ponto importante é o uso de técnicas de melhoramento de imagens. Em sua maioria, os trabalhos apenas fazem extração de características seguido de um classificador, sem se preocupar em pré-processar as imagens para destacar características específicas das imagens. Neste trabalho foi proposto um conjunto de técnicas de processamento de imagens para destacar características das imagens microscópicas de glóbulos brancos.

Além disso, observa-se que a utilização de um classificador interfere diretamente na classificação, portanto, há uma grande diversidade na escolha desses classificadores. Observando a literatura, o método proposto aliou as abordagens de extração de característica e optou por não utilizar um modelo de aprendizado profundo devido ao grande custo computacional exigido. Assim, foi introduzido o uso da XGBoost proposto por [Chen et al. 2015], que é um algoritmo que apresenta forte desempenho em termos de velocidade, escalabilidade e consumo de recursos de hardware.

\section{Materiais e Método}

O método proposto é composto por cinco etapas. A primeira etapa é a aquisição da base de dados, onde utilizou-se uma base pública e disponível no Kaggle. Em seguida, são aplicadas técnicas de processamento de imagens para realçar a textura das imagens, melhorando a distinção entre as classes. Na terceira etapa, é feita a detecção dos glóbulos brancos através de técnica de limiarização. Na quarta etapa, são extraídas as características de textura e forma, propondo o uso do algoritmo TAS em imagens de glóbulos brancos. Por fim, é construído um modelo preditivo usando o XGBoost e 
métricas de validação são calculadas para mensurar a robustez do método. A Figura 1 ilustra o fluxo do método proposto.

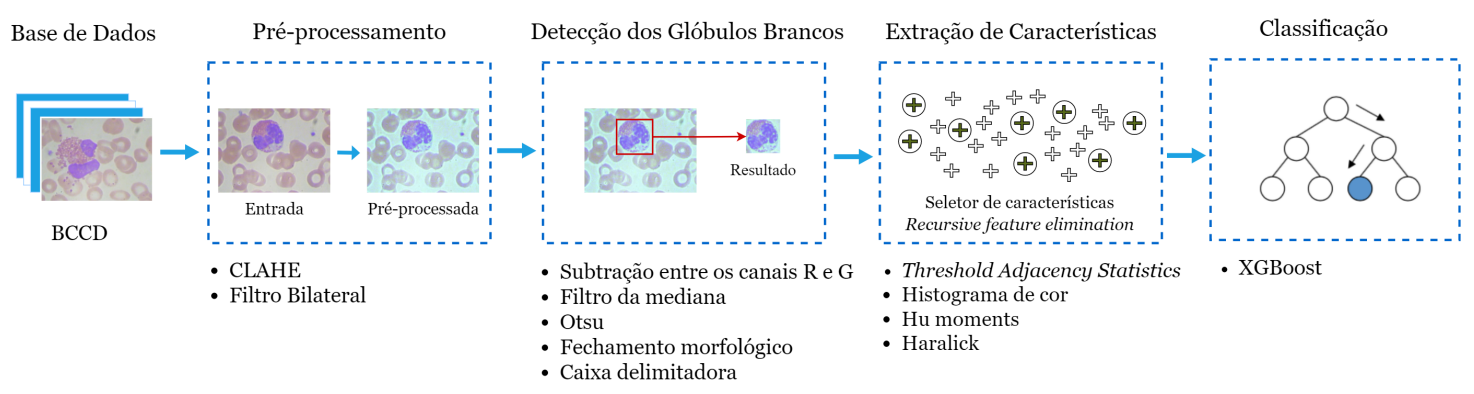

Figura 1. Fluxo do método proposto.

\subsection{Base de Dados}

Para desenvolver o método, foi necessário obter imagens microscópicas de uma base de dados público. Desta forma, seria possível criar um método abrangente e comparável à literatura. Para isto, foi usada a base de dados BCCD [BCCD 2018] do desafio Kaggle, composta por 410 imagens no formato JPG com quatro classes a serem consideradas: 88 eosinófilos, 33 linfócitos, 21 monócitos e 207 neutrófilos. As imagens desta base têm tamanho de $640 \times 480$ pixels no espaço de cores RGB. A Figura 2 apresenta exemplos das quatro classes da base de dados.

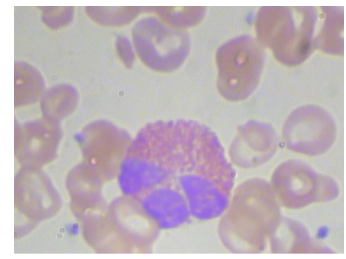

(a) Eosinófilos

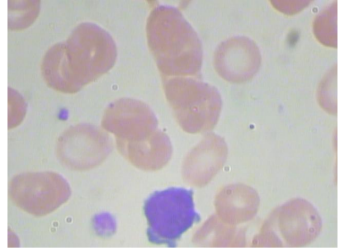

(b) Linfócitos

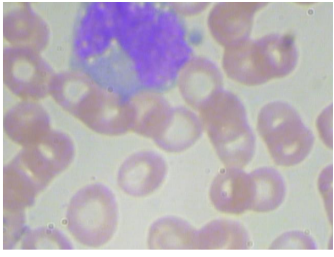

(c) Monócitos

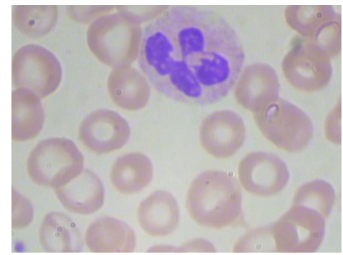

(d) Neutrófilos

Figura 2. Categorias das glóbulos brancos.

\subsection{Pré-processamento}

Ao observar a literatura (Seção 2), não encontra-se estudos que se preocuparam em usar qualquer melhoria de imagem para destacar as imagens dos glóbulos brancos e, consequentemente, aumentar a discriminação entre as classes. Assim, propõe-se um pré-processamento que visa realçar as imagens para melhorar a precisão da extração de características e classificação.

Para a etapa de pré-processamento, propõe-se o usa da sequência de dois filtros. O primeiro filtro é o Contrast-Limited Adaptive Histogram Equalization (CLAHE) [Gonzalez and Woods 2010] que cria uma imagem com níveis de cinza igualmente distribuídos, destacando as características mais marcantes em cada classe e criando uma melhor distinção entre as demais, para isso, foi aplicado em cada um dos canais RBG. O segundo filtro é bilateral, este busca suavizar as imagens preservando as bordas, por meio de uma combinação não linear de valores próximos à 


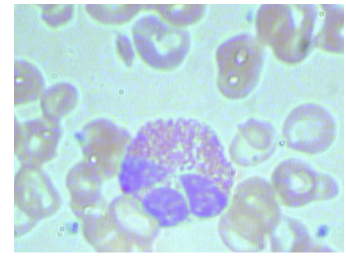

(a) Eosinófilos

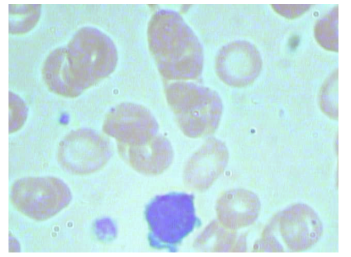

(b) Linfócitos

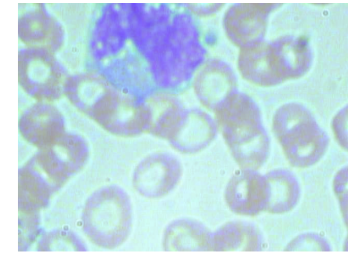

(c) Monócitos

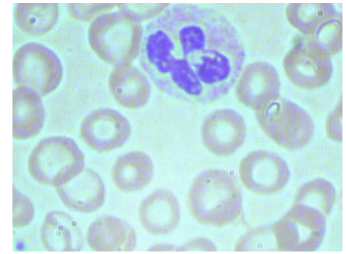

(d) Neutrófilos

Figura 3. Resultado do pré-processamento aplicado as imagens da Figura 2.

imagem [Wu et al. 2007]. Um exemplo de cada uma das classes com a etapa de préprocessamento é mostrado na Figura 3.

Observando as imagens, destaca-se que os filtros foram capazes de realçar de forma efetiva os padrões das imagens microscópicas.

\subsection{Detecção de Glóbulos Brancos}

Como as imagens são de tamanho $640 \times 480$ e contêm mais informações além dos glóbulos (Figura 4 (a)), sugere-se uma etapa de detecção dos glóbulos brancos. A região relevante da imagem é caracterizada pela tonalidade lilás (combinação do vermelho com azul), assim, apenas os canais R e G da imagem de entrada em RGB são considerados, sendo feita uma subtração entre eles (Figura 4 (b)). Posteriormente, o filtro da mediana [Gonzalez and Woods 2010] (Figura 4 (c)) é aplicado para remover informações irrelevantes (ruídos), seguido pelo uso do Otsu [Sezgin and Sankur 2004] (Figura 4 (d)), que é uma técnica de limiarização usada para localizar o núcleo celular, uma vez que o núcleo possui uma pigmentação que o distingue dos demais elementos da imagem. A operação morfológica de fechamento [Gonzalez and Woods 2010] (Figura 4 (e)) é adicionada para restabelecer as conexões sem modificar radicalmente o tamanho e a forma inicial do corpo celular, usando o elemento estruturante retangular de tamanho cinco. Finalmente, é necessário identificar todo o corpo celular. Para isso, o maior núcleo celular é encontrado e uma caixa delimitadora com um espaço de 10 pixels (Figura 4 (f)) é criada (este espaço foi adicionado para ter uma certa margem a fim de inserir as características das bordas entre o glóbulo e demais estruturas). A Figura 4 ilustra um exemplo seguindo as etapas da detecção de glóbulos brancos.

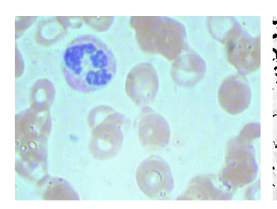

(a) Imagem de entrada

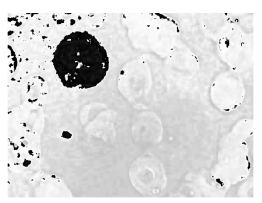

(b) Subtração entre
os canais R e G

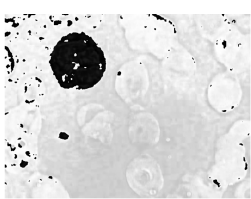

(c) Filtro da mediana

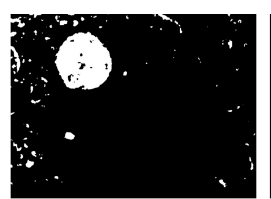

(d) Otsu

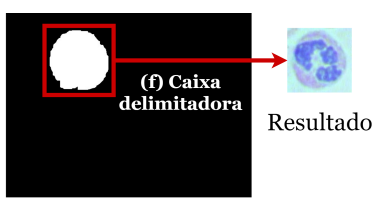

(e) Fechamento morfológico

Figura 4. Etapas da detecção de glóbulos brancos.

Em todos os testes experimentais, foram aplicados a etapa de detecção de glóbulos brancos. Com as imagens da detecção de glóbulos brancos criadas, a próxima etapa é a extração de características.

\subsection{Extração de Características}

Esta etapa é realizada para extrair características que são robustas em discriminar os dados de interesse [Meyer-Baese and Schmid 2014], que devem ser informativas e não 
redundantes para que o modelo tenha um alto desempenho na diferenciação das classes. Para a etapa de extração de características, quatro grupos foram usados neste trabalho:

1. TAS [Hamilton et al. 2007]: em essência, a ideia principal do TAS é definir um limiar de uma imagem e contar o número de pixels acima do limiar com um determinado número de pixels adjacentes. Isso serve para distinguir e classificar imagens de diferentes localizações subcelulares. Em comparação a outras estatísticas comumente usadas, o TAS tem uma ordem de magnitude mais rápida para calcular, enquanto fornece alta precisão de classificação. O limiar aplicado foi calculado automaticamente usando o Otsu, e um conjunto de 162 características foi obtido.

2. Histograma de cor [Gonzalez and Woods 2010]: é uma técnica comum usada para representar o conteúdo das cores nas imagens. Inicialmente, um espaço de cores é selecionado para a representação das características das cores. Como o espaço de cores RGB não é perceptualmente uniforme, ou seja, a distância de cores em RGB não corresponde à dissimilaridade de cores na percepção, os dados da imagem foram transformados no espaço de cores HSV. Posteriormente, a distribuição do histograma para cada um dos canais HSV foi obtida. Finalmente, foi retornado um conjunto de 512 características.

3. Hu Moments [Gonzalez and Woods 2010]: são um conjunto de 7 características muito utilizadas em processamento de imagens. Elas são calculadas usando momentos centrais que são invariantes às transformações da imagem. Os primeiros 6 momentos provaram ser invariantes à translação, escala, rotação e reflexão. Enquanto o sinal do sétimo momento muda para reflexão da imagem.

4. Haralick [Gonzalez and Woods 2010]: essa técnica é baseada nas distribuição de probabilidades conjuntas em pares de pixels e consiste em 13 características. Para isso, são usados as matrizes de coocorrência de níveis de cinza (Gray-Level Cooccurrence Matrix - GLCM), que mostra a frequência com que cada nível de cinza ocorre em um pixel localizado em uma posição geométrica fixa em relação a cada um dos outros pixels, em função do nível de cinza. Posteriormente, são calculados os descritores de Haralick baseados no GLCM.

Vale destacar que o TAS foi proposto para classificação de imagens de proteínas subcelular [Hamilton et al. 2007]. No trabalho proposto, é a primeira vez que é utilizado em conjunto com características de textura e forma para classificar glóbulos brancos em imagens microscópicas.

\subsubsection{Seleção de Características}

Por serem extraídas 694 características e para evitar a redundância entre elas, sugere-se a etapa de seleção de características. O algoritmo Recursive Feature Elimination (RFE) foi proposto por [Guyon et al. 2002] para seleção de genes no problema de classificação usando Máquinas de Vetores de Suporte. De acordo com [Guyon et al. 2002], dado um estimador externo, que pesa as características de um problema (pode ser SVM, Regressor Linear, entre outros como XGBoost neste caso), o objetivo do RFE é selecionar as características recursivamente considerando subconjuntos menores reduzindo o subconjunto atual. 
Resumidamente, o estimador externo é treinado com um conjunto inicial de características e a importância de cada característica é obtida atribuindo um coeficiente para cada uma. As características menos importantes são removidas do conjunto atual de características. Este procedimento é repetido recursivamente no conjunto até que o número desejado de características a serem selecionadas seja atingido [Guyon et al. 2002].

Vale lembrar que, por se tratar de uma eliminação recursiva e iterativa, é necessário que haja um parâmetro que indique o número final de características a serem escolhidas. Para contornar o problema de parametrização, $10 \%$ da base de treinamento foi separada e a cada iteração do algoritmo, as métricas foram validadas nesta base separada. O algoritmo parou a execução quando não houve melhoria nas métricas.

\subsection{Classificação - XGBoost}

Com características extraídas e selecionadas, a próxima etapa classifica as imagens microscópicas entre as quatro classes. Neste trabalho foi utilizado o classificador XGBoost, devido ao seu forte desempenho (em termos de velocidade, escalabilidade, consumo de memória e recursos de hardware [Chen et al. 2015]) em comparação com outras soluções existentes.

O XGBoost consiste na biblioteca proposta em [Chen et al. 2015] baseada no framework de aumento de gradiente desenvolvido por [Friedman 2001]. Pode ser usado para várias funções objetivo, incluindo regressão e classificação. O XGBoost oferece uma implementação eficiente e escalonável do algoritmo de árvore de decisão baseado em gradiente. Dessa forma, foi amplamente aplicado e sua eficiência foi reconhecida em vários desafios de aprendizado de máquina e mineração de dados (por exemplo, o site Kaggle), alcançando resultados de última geração para uma ampla gama de problemas [Chen et al. 2015].

O XGBoost é extensível e seus parâmetros podem ser facilmente alterados [Carvalho et al. 2020]. Seus parâmetros incluem max depth, learning rate, gamma, colsample by tree, min child weight e fitness. Neste estudo, foram usados os parâmetros padrões definidos por [Chen et al. 2015], detalhes do algoritmo podem ser vistos em seu trabalho.

Após a criação do modelo XGBoost, métricas de validação foram extraídas. Neste trabalho, foram utilizadas as métricas acurácia, precisão, sensibilidade e F-Measure.

\section{Resultados e Discussão}

Nesta seção, é apresentado o ambiente de treinamento, o resultado de cada etapa, o desempenho do método no estudo de caso e, por fim, a comparação com a literatura.

\subsection{Ambiente de Treinamento}

O método foi implementado usando a biblioteca Python. Utilizou-se principalmente a biblioteca de aprendizado profundo Keras [Chollet et al. 2015] com TensorFlowGPU [Abadi et al. 2015] como back-end. Nos experimentos o computador usado consiste em uma CPU Intel Core i7-7700K de 4,20 GHz, 16 GB de RAM e placa de vídeo Nvidia GeForce GTX 1080-Ti, rodando em um sistema operacional Windows 10. 


\subsection{Resultados do Método Proposto}

Primeiramente, todas as imagens passaram pelas etapas de pré-processamento (Seção 3.2) e detecção de glóbulos brancos (Seção 3.3), que resultou em imagens realçadas e apenas a região de interesse a ser classificada. Em seguida, as imagens passaram pela etapa de extração de características onde foram aplicadas as técnicas descritas na Seção 3.4. Por fim, a base de dados foi dividida aleatoriamente em $80 \%$ de cada classe para o treinamento (total de 243 imagens) e $20 \%$ para teste (total de 104 imagens), onde foi criado um modelo pelo XGBoost e aplicado a base de teste e as métricas extraídas (Seção 3.5). Nas próximas subseções são apresentados experimentos para verificar a efetividade do método, evidenciando todas as etapas que o compõe.

\subsubsection{Experimento 1 - Validação da Etapa de Pré-processamento}

Primeiramente, para validar a influência do pré-processamento executou-se o método completo com e sem essa etapa. Os resultados são apresentados na Tabela 1.

Tabela 1. Resultados do método avaliando a etapa de pré-processamento.

\begin{tabular}{ccccc}
\hline Técnica & Acc (\%) & Precisão (\%) & Sensibilidade (\%) & F-Measure \\
\hline Sem pré-processamento & 89,42 & 91 & 84 & 87 \\
\hline Com pré-processamento & 93,27 & 93 & 93 & 90 \\
\hline
\end{tabular}

Embora os resultados sem esta etapa já serem bastante promissores, com métricas acima de $84 \%$, pode-se perceber que a etapa de pré-processamento é muito promissora, alcançando uma melhoria de mais de $2 \%$ em todas as métricas com a aplicação dos filtros CLAHE e bilateral (ênfase na acurácia que passou de 89,42\% para 93,27\%). Acredita-se que ao realçar as estruturas dos glóbulos brancos da base de dados, houve uma maior distinção entre as classes e facilitou os extratores de características na tarefa de diferenciação.

\subsubsection{Experimento 2 - Validação da Etapa de Extração de Características}

No segundo experimento conduzido, avaliou-se os quatro grupos de características separadamente a fim de observar a influência de cada uma para o modelo. A Tabela 2 apresenta os resultados deste experimento.

Tabela 2. Resultados do método avaliando a etapa de extração de características.

\begin{tabular}{lcccc}
\hline \multicolumn{1}{c}{ Características } & Acc (\%) & Precisão (\%) & Sensibilidade (\%) & F-Measure \\
\hline TAS & 87,50 & 88 & 88 & 87 \\
Histograma de Cor & 81.73 & 82 & 82 & 82 \\
Haralick & 65,38 & 64 & 65 & 61 \\
Hu Moments & 50 & 39 & 50 & 40 \\
\hline Concatenadas+RFE & 93,27 & 93 & 93 & 90 \\
\hline
\end{tabular}

Observa-se que o TAS e o histograma de cor apresentam as melhores métricas individuais. Destaca-se que ambas são as que apresentam o maior número de 
características, desta forma conseguem fazer uma maior representatividade do conjunto de amostras. Embora os extratores HU Moments e Haralick sejam bem conhecidos, isoladamente não são capazes de obter resultados expressivos. Porém, ao combinar os quatro conjuntos de características, os resultados alcançados são aprimorados, apresentando resultados superiores a $90 \%$ em todas as métricas.

Ainda, para avaliar o uso do RFE como seletor de características, apresenta-se na Tabela 3 os resultados comparando o método com e sem o seletor. O RFE apresentado na Seção 3.4.1 selecionou 194 características do conjunto de 694, sendo 116 de TAS, 68 de Histograma de cor, 6 de Hu Moments e 4 de Haralick. É importante observar que dentre os extratores, o TAS obteve mais características selecionadas, demonstrando que produz medidas altamente descritivas das categorias.

Tabela 3. Resultados do método avaliando a etapa de seleção de características.

\begin{tabular}{lcccc}
\hline Seleção de Características & Acc (\%) & Precisão (\%) & Sensibilidade (\%) & F-Measure \\
\hline Sem RFE & 91,35 & 91 & 91 & 91 \\
\hline Com RFE & 93,27 & 93 & 93 & 90 \\
\hline
\end{tabular}

Percebe-se que ao selecionar as melhores características dos quatro grupos, obtém-se uma melhora de $2 \%$ em todas as métricas e as características são reduzidas de 694 para 194, evitando redundância entre as características extraídas.

\subsubsection{Experimento 3 - CNNs vs Método Proposto}

Finalmente, nos últimos anos com os avanços dos hardwares e a possibilidade de execução de redes neurais profundas, os resultados destas redes têm mostrado resultados surpreendentes em diversos domínios [Diniz et al. 2020, da Cruz et al. 2020]. Para comparar o método proposto com as CNNs, duas redes foram treinadas (VGG19 e ResNet50). Para isso, as etapas de extração e classificação do método foram substituídas pela VGG-19 e ResNet50. As configurações das redes foram feitas conforme propostas no trabalho de [Vatathanavaro et al. 2018] com 100 épocas cada, usando os pesos prétreinados aplicados a base de dados ImageNet. A Tabela 4 apresenta os resultados deste experimento.

Tabela 4. Resultados do método comparando com CNNs.

\begin{tabular}{lcccc}
\hline \multicolumn{1}{c}{ Classificador } & Acc (\%) & Precisão (\%) & Sensibilidade (\%) & F-Measure \\
\hline VGG19 & 75 & 76 & 75 & 73 \\
Resnet50 & 74,04 & 74 & 74 & 74 \\
\hline Método Proposto & 93,27 & 93 & 93 & 90 \\
\hline
\end{tabular}

Embora essas redes abstraiam as etapas de engenharia características, elas não foram capazes de ser eficazes na classificação dos glóbulos brancos. Acredita-se que, para esta tarefa, as CNNs não foram robustas devido à pequena quantidade de amostras para cada classe. Além disso, a CNN sozinha tem um alto custo computacional, e as técnicas de aumento de dados poderiam melhorar sua performance, mas aumentaria ainda mais o custo. Portanto, no método proposto, foi usado o XGBoost que, ao contrário das CNNs, é uma técnica rápida, com pouco consumo de recursos computacionais e forte desempenho. 


\subsection{Comparação com a Literatura}

Nesta seção apresenta uma comparação com os trabalhos relacionados descritos na Seção 2. A Tabela 5 mostra essa comparação.

Tabela 5. Comparação com os trabalhos relacionados.

\begin{tabular}{lcccc}
\hline Método & Acc (\%) & Precisão (\%) & Sensibilidade (\%) & F-Measure \\
\hline [Rezatofighi and Soltanian-Zadeh 2011] & 93,09 & - & - & - \\
[Ko et al. 2011] & - & 72,5 & 67,34 & - \\
[Tai et al. 2011] & - & 95,27 & 95,3 & - \\
[Macawile et al. 2018] & 95 & - & - & - \\
[Vatathanavaro et al. 2018] & 88,29 & - & - & - \\
[H Mohamed et al. 2020] & 92 & 86 & & 86 \\
\hline Método Proposto & 93,27 & 93 & 93 & 90 \\
\hline
\end{tabular}

Observa-se que os trabalhos de [Rezatofighi and Soltanian-Zadeh 2011, Ko et al. 2011, Tai et al. 2011] propuseram abordagens tradicionais para extrair características. O método proposto neste trabalho apresenta uma nova abordagem usando descritores de textura e forma e o classificador XGBoost e apresentou resultados comparáveis a esses estudos.

Por outro lado, os trabalhos que utilizam técnicas de aprendizado profundo [Macawile et al. 2018, Vatathanavaro et al. 2018, H Mohamed et al. 2020] são importantes por extraírem características por meio das redes, mas nem sempre essas características são suficientemente representativas para solucionar o problema. Apenas o trabalho de [Macawile et al. 2018] apresentou resultados superiores ao método proposto neste artigo. Todavia, a base de dados usada contém apenas 178 imagens divididas em 5 classes, enquanto no método proposto usou mais de 400, i.e., o dobro. Dessa forma, destaca-se que o método proposto, além de inovador e apresentar resultados promissores, ocupa lugar de destaque na literatura.

\subsection{Estudos de Caso}

Nesta seção, são descritos estudos de caso do método. Após o modelo criado, as imagens de teste foram aplicadas, e os resultados de acerto e erro são mostrados na Figura 5.
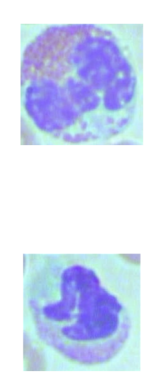

(a) Eosinófilos
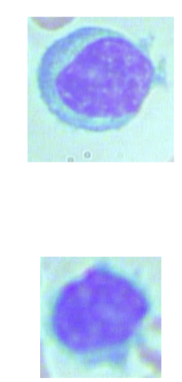

(b) Linfócitos

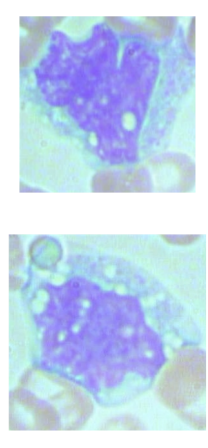

(c) Monócitos
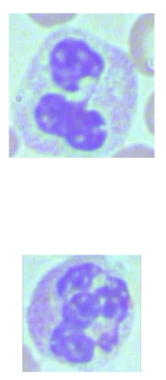

(d) Neutrófilos

Figura 5. Estudos de caso: primeira linha imagens classificadas corretamente, segunda linha imagens classificadas erroneamente.

Observa-se, na primeira linha, que o método foi eficaz em classificar corretamente as quatro classes, que apesar de parecerem visualmente semelhantes, dadas suas 
características extraídas de textura e forma, puderam ser diferenciadas pelo método proposto. Porém, na segunda linha, percebe-se que o método errou alguns casos, o que também é confirmado pelo fato da grande semelhança entre as imagens microscópicas, resultando em uma acurácia de 93,27\%.

\section{Conclusões}

Neste trabalho foi apresentado um método automático para classificação de glóbulos brancos. Uma de suas principais vantagens é que possui um custo computacional consideravelmente baixo, comparado às abordagens de aprendizado profundo, uma vez que usa características de textura e formas extraídas da imagem ao invés de todo treinamento com CNNs. Ainda, foram propostas técnicas de realce de imagens que, como destacado nos resultados, mostraram-se promissoras para auxiliar as demais etapas do método. Além do uso do extrator TAS de forma efetiva e do classificador XGBoost, que apresenta um bom desempenho comparado as CNNs.

Como trabalhos futuros, pretende-se analisar outras técnicas de textura, além de descritores de forma, como LBP, HOG, entre outros. Por fim, explorar alternativas relacionadas à detecção dos glóbulos brancos usando redes de segmentação. Acredita-se que as modificações mencionadas possam proporcionar melhores resultados.

\section{Referências}

Abadi, M., Agarwal, A., Barham, P., Brevdo, E., Chen, Z., Citro, C., Corrado, G. S., Davis, A., Dean, J., Devin, M., et al. (2015). Tensorflow: Large-scale machine learning on heterogeneous systems. Software available from tensorflow.org.

ACS (2018). About chronic lymphocytic leukemia. https://cancer.org/cancer/chroniclymphocytic-leukemia/about/normal-tissue.html. Acesso em: 2020-06-07.

BCCD, D. (2018). Bccd dataset. https://www.kaggle.com/surajiiitm/bccd-dataset.

Carvalho, E. D., Antônio Filho, O., Silva, R. R., Araújo, F. H., Diniz, J. O., Silva, A. C., Paiva, A. C., and Gattass, M. (2020). Breast cancer diagnosis from histopathological images using textural features and cbir. Artificial Intelligence in Medicine, 105:101845.

Chen, T., He, T., Benesty, M., Khotilovich, V., and Tang, Y. (2015). Xgboost: extreme gradient boosting. R package version $0.4-2$, pages $1-4$.

Chollet, F. et al. (2015). Keras. https://keras.io.

da Cruz, L. B., Araújo, J. D. L., Ferreira, J. L., Diniz, J. O. B., Silva, A. C., de Almeida, J. D. S., de Paiva, A. C., and Gattass, M. (2020). Kidney segmentation from computed tomography images using deep neural network. Computers in Biology and Medicine, page 103906.

Diniz, J. O. B., Ferreira, J. L., Diniz, P. H. B., Silva, A. C., and de Paiva, A. C. (2020). Esophagus segmentation from planning ct images using an atlas-based deep learning approach. Computer Methods and Programs in Biomedicine, 197:105685.

Friedman, J. H. (2001). Greedy function approximation: A gradient boosting machine. The Annals of Statistics, 29(5):1189-1232.

Gonzalez, R. C. and Woods, R. E. (2010). Digital Image Processing. Pearson Prentice Hall. 
Guyon, I., Weston, J., Barnhill, S., and Vapnik, V. (2002). Gene selection for cancer classification using support vector machines. Machine learning, 46(1-3):389-422.

H Mohamed, E., H El-Behaidy, W., Khoriba, G., and Li, J. (2020). Improved white blood cells classification based on pre-trained deep learning models. Journal of Communications Software and Systems, 16(1):37-45.

Hamilton, N. A., Pantelic, R. S., Hanson, K., and Teasdale, R. D. (2007). Fast automated cell phenotype image classification. BMC bioinformatics, 8(1):1-8.

Ko, B., Gim, J., and Nam, J. (2011). Cell image classification based on ensemble features and random forest. Electronics Letters, 47(11):638-639.

Macawile, M. J., Quiñones, V. V., Ballado, A., Cruz, J. D., and Caya, M. V. (2018). White blood cell classification and counting using convolutional neural network. In $20183 \mathrm{rd}$ International conference on control and robotics engineering (ICCRE), pages 259263. IEEE.

Meyer-Baese, A. and Schmid, V. (2014). Chapter 2 - feature selection and extraction. In Meyer-Baese, A. and Schmid, V., editors, Pattern Recognition and Signal Analysis in Medical Imaging (Second Edition), pages 21 - 69. Academic Press, Oxford, second edition edition.

Mohamed, E., El-Behaidy, W., Khoriba, G., and Li, J. (2020). Improved white blood cells classification based on pre-trained deep learning models. Journal of Communications Software and Systems, 16.

NCI (2020). White blood cell. https://cancer.gov/publications/dictionaries/ cancerterms/def/white-blood-cell. Acesso em: 2020-06-07.

Rezatofighi, S. H. and Soltanian-Zadeh, H. (2011). Automatic recognition of five types of white blood cells in peripheral blood. Computerized Medical Imaging and Graphics, 35(4):333-343.

Sezgin, M. and Sankur, B. (2004). Survey over image thresholding techniques and quantitative performance evaluation. Journal of Electronic imaging, 13(1):146-165.

Tai, W.-L., Hu, R.-M., Hsiao, H. C., Chen, R.-M., and Tsai, J. J. (2011). Blood cell image classification based on hierarchical svm. In 2011 IEEE International Symposium on Multimedia, pages 129-136. IEEE.

Vatathanavaro, S., Tungjitnob, S., and Pasupa, K. (2018). White blood cell classification: A comparison between vgg-16 and resnet-50 models. In Thailand-CIS, page 3.

Vogado, L. H., Veras, R. M., Araujo, F. H., Silva, R. R., and Aires, K. R. (2019). Rede neural convolucional para o diagnóstico de leucemia. In Anais do XIX Simpósio Brasileiro de Computação Aplicada à Saúde, pages 46-57. SBC.

Wu, Y.-T., Wei, J., Hadjiiski, L. M., Sahiner, B., Zhou, C., Ge, J., Shi, J., Zhang, Y., and Chan, H.-P. (2007). Bilateral analysis based false positive reduction for computer-aided mass detection. Medical physics, 34(8):3334-3344.

Zhao, J., Zhang, M., Zhou, Z., Chu, J., and Cao, F. (2017). Automatic detection and classification of leukocytes using convolutional neural networks. Medical \& biological engineering \& computing, 55(8):1287-1301. 